\section{Mit nur vier Injektionen im Vorteil}

$\mathrm{B}$ ei der spezifischen Immuntherapie (SIT) spielt neben der Wirksamkeit und Sicherheit der Behandlung auch die Praktikabilität eine wichtige Rolle. Prof. Dr. Wolfgang Czech, Villingen-Schwenningen, machte darauf aufmerksam, dass eine effiziente SIT nicht nur für den $\mathrm{Pa}$ tienten interessant ist, sondern auch für den behandelnden Arzt - vor allem im Rahmen der vieldiskutierten Regelleistungsvolumina. Eine solche KomplettTherapie mit nur vier Injektionen ist mit POLLINEX $^{\circledR}$ Quattro möglich.

Ein wesentlicher Aspekt bei immunmodulierenden Substanzen ist das im Präparat enthaltende Adjuvans, das die Immunantwort auf die Antigene verstärkt. Dr. Jens Vollmar vom Unternehmen GlaxoSmithKline, München, erläuterte die Vorteile des patentierten Adju- vans $\mathrm{MPL}^{\circledR}$, das auch in POLLINEX ${ }^{\circledR}$ Quattro enthalten ist. Mit MPL ${ }^{\circledR}$ adjuvantierte Vakzinen führen zu einer verbesserten Immunantwort verglichen mit solchen Impfstoffen, die nur mit Aluminiumsalzen adjuvantiert sind. MPL ${ }^{\circledR}$ ist unter anderem auch im aktuellen HPVImpfstoff von GlaxoSmithKline enthalten. In der spezifischen Immuntherapie ist POLLINEX ${ }^{\circledR}$ Quattro das einzige Präparat mit dem Adjuvans $\mathrm{MPL}^{\circledR}$.

Die Anforderungen an klinische Studien steigen auch in der Allergologie rapide an. Prof. Dr. Werner Aberer aus Graz, Österreich, verwies auf die neuen strengen Vorgaben der GCP/ICH-Richtlinie. „Die POLLINEX ${ }^{\circledR}$ Quattro-G301Studie als weltweit größte kontrollierte und erfolgreiche Studie auf dem Gebiet der spezifischen Immuntherapie erfüllt

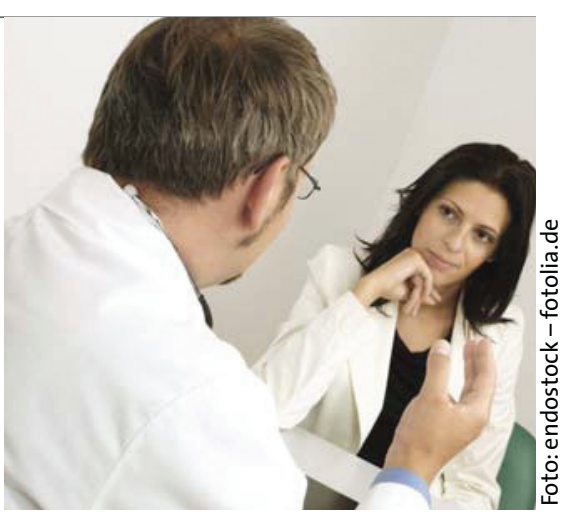

Viele Patienten legen Wert auf eine Immuntherapie mit wenig Injektionen.

diese hohen Ansprüche." Einmal mehr werden damit Wirksamkeit und Sicherheit dieser Kompletttherapie mit nur vier Injektionen, die in Deutschland bereits seit zehn Jahren verfügbar ist, belegt. af

Industriesymposium „Komplett und flexibel: Die Immuntherapie mit vier Injektionen \& Wie viel SIT kommt beim Patienten an?".4. Gemeinsamer Deutscher Allergiekongress, Berlin, 3.-6. September 2009. Veranstalter: Bencard, München

\title{
Immuntherapie sorgt für „well days“
}

W irksamkeit und Verträglichkeit einer präsaisonalen subkutanen Immuntherapie (SCIT) mit dem SechsGräserpollen-Allergoid Allergovit ${ }^{\circledR}$ sind bereits mehrfach belegt. Dr. Annemie Narkus, Reinbek, stellte erstmals auch Daten zur Langzeitwirkung bei Erwachsenen vor: Bei Patienten mit Gräserpollenallergie klagten die Probanden der Verumgruppe auch drei Jahre nach Abschluss einer SCIT mit Allergovit ${ }^{\circledR}$ über deutlich weniger Augenjuckreiz, Nasenfluss und bronchiale Symptome als die Probanden der Plazebogruppe. Dementsprechend fühlten sie sich weniger durch allergische Symptome belastet: An 39 von 42 Tagen in der Gräserpollenflugzeit gaben sie einen Symptomscore von vier oder weniger an, ohne dass eine Medikamenteneinnahme nötig gewesen war was einem so genannten „well day“ entspricht. In der Kontrollgruppe dagegen galt dies nur für ein Drittel der Tage. Auch die Rate an neuen Sensibilisierungen war unter der SCIT deutlich geringer. Zu diesem Ergebnis war auch Dr. Peter Eng, Aarau, Schweiz, gekommen, der die Daten von Kindern nach dem Ende einer SCIT mit Allergovit ${ }^{\circledR}$ zwölf Jahre lang weiter dokumentiert hatte.
Für Prof. Dr. J. Christian Virchow, Rostock, stellt die spezifische Immuntherapie als kausaler Therapieansatz der allergischen Rhinitis gleichzeitig ein wichtiges Mittel zur Prävention von Asthma dar. Von einer „Erfolgsgeschichte" sprach Kongresspräsident Prof. Dr. Ulrich Wahn, Berlin, und betonte dabei mit Blick auf die von der europäischen Zulassungsbehörde EMEA formulierten Ziele: „Kein anderes Medikament ist je angetreten, Allergie zu heilen." bv

Industriesymposium „Erfolgsfaktoren für eine innovative SIT“. 4. Gemeinsamer Deutscher Allergiekongress, Berlin, 3.-6. September 2009. Veranstalter: Allergopharma, Reinbek

\section{$\mathrm{Al}(\mathrm{OH})_{3}$ - vom Vehikel zum Adjuvans}

$\mathrm{M}$ enschen mit wenig Zeit sind froh, wenn die Aufdosierungsphase einer subkutanen Immuntherapie möglichst kurz ist. Durch neue Ergebnisse aus der immunologischen Grundlagenforschung kann dieser Wunsch jetzt auch von ALK-Abelló erfüllt werden: Es hat sich nämlich gezeigt, dass sich durch eine Veränderung des Verhältnisses von Aluminiumhydroxid zu Allergen die Immunogenität der Allergene deutlich steigern lässt. Diese Beobachtung wird nun mit dem neuen Präparat Avanz ${ }^{\circledR}$ umgesetzt: Das Allergen-Adjuvans-Verhältnis be- trägt in Avanz ${ }^{\circledR} 1: 3$ statt wie bisher $1: 1$, zugleich konnte das Injektionsvolumen von $1 \mathrm{ml}$ auf $0,5 \mathrm{ml}$ reduziert werden. „Durch den Aluminiumturbo kann die Gesamtdosis von Allergen plus Adjuvans im Vergleich zu ALK-Depot $S Q^{\circledR}$ deutlich gesenkt werden“, erklärte Prof. Dr. Ludger Klimek, Wiesbaden.

Die Aufdosierung ist bei Avanz ${ }^{\circledR}$ jetzt mit nur fünf Injektionen in vier 